\title{
La migración invisible de las mujeres mexicanas cualificadas
}

\author{
Sara Salvatori' (iD 0000-0002-2298-4472 \\ Teresa Terrón-Caro ${ }^{2}$ (D) 0000-0003-4177-0346 \\ 'Scalabrini International Migration Institute, Roma, RM, Italia. \\ C.P. 00153 -segreteria@simiroma.org \\ 2Universidad Pablo de Olavide. Facultad de Ciencias de la Educación. \\ Departamento de Educación y Psicología Social, Sevilla, España. \\ C.P.41013-mttercar@upo.es
}

\section{$-$}

Resumen: El artículo analiza, desde una perspectiva basada en el análisis del género y la clase social, los mecanismos que determinan la invisibilización de las mujeres mexicanas cualificadas en los flujos migratorios hacia Estados Unidos. Para ello, se utilizan los datos cualitativos que hemos recaudado durante el trabajo de campo realizado en Monterrey (México) y Houston (Estados Unidos) entre 20082011 y en 2016. Los resultados de la investigación apuntan a la coexistencia de invisibilidades que, si por un lado se relacionan a la sobrerrepresentación de los varones en los flujos migratorios, por otro tienen que ver con el desclasamiento experimentado por las mujeres cualificadas en la sociedad de asentamiento. De este modo, las estrategias migratorias evidenciadas a partir del análisis cualitativo adquieren el carácter de mecanismos de resistencia ante los desafíos impuestos a las mujeres por el macrosistema.

Palabras clave: mujeres; mexicanas; cualificadas; migración; mercado de trabajo.

\section{A migração invisível das mulheres mexicanas qualificadas}

Resumo: O artigo analisa, numa perspectiva baseada na análise de gênero e classe social, os mecanismos que determinam a invisibilidade de mulheres mexicanas qualificadas nos fluxos migratórios para os Estados Unidos. Para isso, são utilizados os dados qualitativos que coletamos durante o trabalho de campo realizado em Monterrey (México) e Houston (Estados Unidos) entre 2008-2011 e 2016. Os resultados da pesquisa apontam para a coexistência de invisibilidades que, se por um lado, se relacionam com a sobrerrepresentação dos homens nos fluxos migratórios, por outro, têm a ver com a desclassificação vivenciada por mulheres qualificadas na sociedade de assentamento. Dessa forma, as estratégias migratórias evidenciadas a partir da análise qualitativa adquirem o caráter de mecanismos de resistência aos desafios impostos às mulheres pelo macrossistema.

Palavras-chave: mulheres; mexicanas; qualificadas; migração; mercado de trabalho.

The Invisible Migration of Skilled Mexican Women

Abstract: The article analyzes, from a perspective based on the analysis of gender and social class, the mechanisms that determine the invisibilization of skilled Mexican women in the migratory flows to the United States. For this, we use the qualitative data we have collected during the field work carried out in Monterrey (Mexico) and Houston (United States) between 2008 and 2011 and in 2016. The results of the research point to the coexistence of invisibilities that if they relate, on the one hand, to the overrepresentation of men in migratory flows, on the other they are linked to the declassification experienced by qualified women in the settlement society. In this way, the migratory strategies evidenced from the qualitative analysis acquire the character of resistance mechanisms to the challenges imposed on women by the macrosystem.

Keywords: Women; Mexican; Skilled; Migration; Labor market. 


\section{Introducción}

A partir de los años 80 del siglo pasado, al lado de los más tradicionales flujos migratorios originarios de las áreas rurales que de México se dirigen a Estados Unidos, se posicionan los movimientos procedentes de las ciudades mexicanas, impulsados por las recurrentes crisis económicas y la saturación de los mercados laborales urbanos (Luis J. SOBRINO, 2010; Fernando LOZANO ASCENCIO; Luciana GANDINI; Telésforo RAMÍREZ GARCÍA, 2015). Las ciudades se convierten así en puertos de salida de las migraciones hacia Estados Unidos, determinando un cambio en la composición de los flujos migratorios que ahora se caracterizan por el aumento del nivel escolar (Raúl DELGADO WISE; Mónica Guadalupe CHÁVEZ ELORZA; Héctor RODRÍGUEZ RAMÍREZ, 2016) y el incremento del número de mujeres involucradas en el desarrollo de proyectos migratorios (Jorge A. BUSTAMANTE, 1994).

Sin embargo, a pesar de estas características, la imagen del varón procedente del campo, con baja escolaridad y un escaso conocimiento del inglés sigue siendo predominante en las representaciones de los movimientos migratorios que se originan desde México, con el resultado de invisibilizar la componente femenina de los desplazamientos (Ofelia WOO MORALES, 2014; CONAPO, 2013; Patricia ARIAS, 2013; Teresa REBOLLEDO GÁMEZ; M. Rocío RODRÍGUEZ CASADO, 2014; Teresa TERRÓN-CARO; María C. MONREAL GIMENO, 2014). Del mismo modo, la cualificación de las mujeres migrantes mexicanas se invisibiliza, favoreciendo la inserción laboral en sectores no calificados de la economía estadounidense.

A partir de estas premisas, en el presente artículo pretendemos dar respuesta a una serie de interrogantes:1) ¿Cuáles son los mecanismos que invisibilizan las mujeres mexicanas cualificadas en los flujos migratorios? 2) ¿Cuáles son las estrategias desarrolladas por las mujeres mexicanas cualificadas para enfrentar los mecanismos que determinan una movilidad social descendente?

Para responder a dichas cuestiones, en la primera parte del artículo reflexionamos acerca de los procesos que, a partir del género y la clase social, determinan el fenómeno de la invisibilización de la componente femenina de los flujos migratorios. Asimismo, mostramos la manera en que estos mecanismos invisibilizadores influyen en el acceso al mercado laboral de las mujeres cualificadas.

En la segunda parte, en cambio, presentamos los resultados de la investigación que se ha realizado durante 2009 y 2016 entre la zona metropolitana de Monterrey (México) y Houston (Texas), enfocándonos en algunos de los testimonios recogidos durante el trabajo de campo. En este contexto, nuestro propósito es el de utilizar las narraciones de las mujeres para reflexionar sobre las dinámicas de inclusión en nichos laborales no calificados y marcados por el género, así como mostrar las estrategias que las mujeres han desarrollado para contrarrestar los efectos de un proceso de movilidad social tendente hacia el descenso.

\section{Método de estudio}

La investigación que se presenta a continuación forma parte del trabajo analítico desarrollado durante la elaboración de la tesis doctoral concluida en 2018 y titulada "La migración invisibilizada de mujeres cualificadas de Monterrey (México) a Houston (Estados Unidos): una interpretación desde el enfoque interseccional".

En este estudio el enfoque cualitativo (Antonio DE LILLO, 2010) marca el diseño de la metodología, cuyo objetivo ha sido el de desarrollar un trabajo intensivo capaz de recoger las vivencias individuales y colectivas de hechos específicos y experiencias humanas. Dentro de las prácticas investigativas se ha optado por la etnográfica (Carlo CAPELLO; Pietro CINGOLANI; Francesco VIETTI, 2014), desarrollando aquellas técnicas que la caracterizan, a saber, la observación participante, la redacción de un diario de campo y la realización de entrevistas semi-estructuradas (Emanuela SALA, 2010). Al mismo tiempo, con el propósito de llevar a cabo una investigación fundamentada en la perspectiva transnacional (Peggy LEVITT; Nina GLICK SCHILLER, 2004; Alejandro PORTES, 2007; Marina ARIZA, 2014; Lynn STEPHEN, 2012), se ha considerado oportuno desarrollar una etnografía multisituada según el planteamiento elaborado por George E. Markus (1995) y Mark A. Falzon (2009). Así pues, bajo esta perspectiva metodológica, el trabajo de campo ha sido realizado en dos contextos geopolíticos diferentes: Monterrey (México) y Houston (Estados Unidos). De todos modos, cabe especificar que contrariamente a la mayoría de los estudios sobre migraciones, el punto de partida de esta investigación ha sido el lugar de origen de los sujetos entrevistados (Carlo CAPELLO; Pietro CINGOLANI; Francesco VIETTI, 2014). De hecho, durante los primeros tres años de trabajo de campo, de 2008 a 201 1, Monterrey ha sido elegida como base desde donde llevar la práctica etnográfica en México y en Estados Unidos.

Diferente es el caso del segundo periodo de trabajo de campo, que se ha desarrollado únicamente en Houston durante el verano de 2016. La elección de esta ciudad norteamericana se da en el marco establecido por Rubén Hernández-León (2008) para el cual Monterrey y Houston forman un espacio social transnacional. En efecto, la intensidad de las conexiones se nos ha 
mostrado no sólo a través de la asiduidad con la cual las y los migrantes visitan a los familiares en México, sino también a través de la frecuencia de los viajes que las y los mexicanos realizan para visitar a los familiares emigrados en Estados Unidos (Camelia TIGAU, 2017).

Ahora bien, cabe especificar que mientras la observación participante y la redacción del diario de campo se han llevado a cabo a partir de 2008 , las entrevistas a las informantes se han realizado solamente a partir de 2009 , cuando las relaciones establecidas en el campo nos han permitido conseguir la confianza necesaria para ahondar en las experiencias de las mujeres. Del total de las entrevistas realizadas durante los dos periodos de trabajo de campo, ${ }^{1}$ se han elegido veintiocho testimonios ${ }^{2}$ procedentes de las mujeres que cumplían con todos los requisitos establecidos para el trabajo de investigación de la tesis doctoral.

Los criterios elegidos para la selección de las informantes son los siguientes: 1) ser mujer; 2) tener estudios universitarios; 3 ) ser originaria de la zona metropolitana de Monterrey o, a causa de la elevada migración interna, ser "regia"3 por adopción; 4) estar desarrollando un proyecto migratorio o haber vuelto a Monterrey tras un periodo en Estados Unidos; 5) haber considerado Houston una etapa importante en la trayectoria migratoria.

A partir de estas características, lo que se destaca es que se trata de mujeres con un elevado nivel de cualificación que se ubican en los estratos medio-altos de la ciudad de Monterrey. En las tablas 1, 2 e 3 mostramos un resumen de las características socioculturales poseídas por las mujeres entrevistadas.

Tabla 1. Características socioculturales de las mujeres entrevistadas

\begin{tabular}{|c|c|c|c|c|c|c|}
\hline Edad & $\begin{array}{c}\text { Lugar de } \\
\text { residencia al } \\
\text { momento de } \\
\text { la entrevista }\end{array}$ & $\begin{array}{c}\text { Estade } \\
\text { civil }\end{array}$ & $\begin{array}{l}\mathrm{N}^{\mathrm{N}} \text { de } \\
\text { hijos }\end{array}$ & Nivel educativo & $\begin{array}{c}\text { Primer empleo } \\
\text { en USA }\end{array}$ & Emples sctual \\
\hline 41 & Houston & Cassda & 0 & $\begin{array}{c}\text { Master en } \\
\text { Informitica de } \\
\text { administracióel }\end{array}$ & Mesera & $\begin{array}{c}\text { Vende } \\
\text { publicidad para } \\
\text { un periódico } \\
\text { latino }\end{array}$ \\
\hline 37 & Houston & Cossida & 2 & $\begin{array}{l}\text { Licenciatura en } \\
\text { Administración }\end{array}$ & $\begin{array}{l}\text { Getette de } \\
\text { restaurante }\end{array}$ & $\begin{array}{c}\text { En American } \\
\text { Ainlines }\end{array}$ \\
\hline 50 & Houston & Cossda & 2 & $\begin{array}{l}\text { Lisenciatura et } \\
\text { Informitica de } \\
\text { administracióel }\end{array}$ & $\begin{array}{c}\text { Cocinabay } \\
\text { vendia tamales }\end{array}$ & $\begin{array}{c}\text { Tiene su propio } \\
\text { negocio }\end{array}$ \\
\hline 42 & Houston & Cossosda & 2 & Mídico & Ama de casa & Ama de casa \\
\hline 37 & Mocterrey & Casada & 2 & $\begin{array}{c}\text { Licenciatura en } \\
\text { Enfermeria }\end{array}$ & Ama de casa & Ama de casa \\
\hline 37 & Houston & Casada & 2 & $\begin{array}{c}\text { Licenciatura en } \\
\text { Administración de } \\
\text { empresas }\end{array}$ & $\begin{array}{c}\text { Empleadia en una } \\
\text { tienda }\end{array}$ & Desempleadi \\
\hline 35 & Monterney & Casada & 1 & $\begin{array}{c}\text { Licenciatura en } \\
\text { Administración de } \\
\text { empresas }\end{array}$ & $\begin{array}{l}\text { Montó una } \\
\text { guardetia } \\
\text { informal en su } \\
\text { piso }\end{array}$ & Secretaria \\
\hline 36 & Houston & Casada & 2 & $\begin{array}{c}\text { Licenciatura en } \\
\text { Administración de } \\
\text { empresas }\end{array}$ & Desempleada & $\begin{array}{c}\text { Conserje en un } \\
\text { botel }\end{array}$ \\
\hline
\end{tabular}

Fuente: elaboración propia a partir de la información recogida en el trabajo de campo de la investigación.

'Entre 2009 y 2011 se ha realizado un total de 50 entrevistas, mientras que en verano de 2016 se han recaudado 20 entrevistas.

${ }_{2}^{2}$ Cabe especificar que las últimas cuatro mujeres que aparecen en la tabla 3 a pesar de no reunir todos los requisitos, han sido incluidas porque son clave en la posibilidad de observar el desarrollo de prácticas transnacionales que caracterizan el contexto urbano.

${ }^{3}$ Este término identifica la zona metropolitana de Monterrey. 
Tabla 2. Características socioculturales de las mujeres entrevistadas

\begin{tabular}{|c|c|c|c|c|c|c|}
\hline 45 & Houston & Casada & 1 & $\begin{array}{l}\text { Licetciatura en } \\
\text { Humanidades }\end{array}$ & Ama de casa & Ama de caka \\
\hline 44 & Manterrey & Soltera & 0 & $\begin{array}{l}\text { Master en } \\
\text { Educsción }\end{array}$ & $\begin{array}{l}\text { Mantenimiento y } \\
\text { limpieza en un } \\
\text { restaurante }\end{array}$ & $\begin{array}{l}\text { Maestra de } \\
\text { ingles }\end{array}$ \\
\hline 37 & Houstan & Soltera & 0 & $\begin{array}{c}\text { Licenciatura en } \\
\text { Pricologia }\end{array}$ & Maestra bilingue & $\begin{array}{l}\text { Maestra } \\
\text { bilingue }\end{array}$ \\
\hline 39 & Houston & Soltera & 0 & $\begin{array}{c}\text { Liceticiatura en } \\
\text { Nutricián }\end{array}$ & Maestra bilingie & $\begin{array}{l}\text { Maectra } \\
\text { bilingue }\end{array}$ \\
\hline 49 & Houston & Soltera & 0 & $\begin{array}{c}\text { Liceteciatura en } \\
\text { Ciencizs } \\
\text { juxidicas }\end{array}$ & $\begin{array}{l}\text { Caidaba una señors } \\
\text { ancians }\end{array}$ & $\begin{array}{l}\text { Maestra } \\
\text { bilingtue }\end{array}$ \\
\hline 41 & Houston & Casada & 1 & $\begin{array}{c}\text { Liceteciatura en } \\
\text { Arquitecturn }\end{array}$ & Msedtra & $\begin{array}{l}\text { Maretra } \\
\text { bilingue }\end{array}$ \\
\hline 41 & Houstan & Casada & 2 & $\begin{array}{c}\text { Licenciatura en } \\
\text { Diseño de } \\
\text { interiores }\end{array}$ & $\begin{array}{l}\text { En la venta de } \\
\text { bienes raices }\end{array}$ & $\begin{array}{l}\text { Maestrn } \\
\text { bilingue }\end{array}$ \\
\hline 47 & Houstan & Casada & 1 & $\begin{array}{l}\text { Lidenciatura en } \\
\text { Contaduria }\end{array}$ & Maestra bilingue & $\begin{array}{l}\text { Masstrn } \\
\text { bilingue }\end{array}$ \\
\hline 50 & Houstan & Casada & 3 & $\begin{array}{c}\text { Lidenciatura en } \\
\text { Educsción }\end{array}$ & Niñena & $\begin{array}{l}\text { Maestra } \\
\text { bilingüe }\end{array}$ \\
\hline 40 & Houston & Soltera & 0 & $\begin{array}{c}\text { Licetciatura en } \\
\text { Ciencias } \\
\text { juxidicas }\end{array}$ & Maestra bilingiee & $\begin{array}{l}\text { Maestra } \\
\text { bilingue }\end{array}$ \\
\hline 58 & Houston & Divorciada & 0 & $\begin{array}{l}\text { Licesciatura en } \\
\text { Idioms }\end{array}$ & Maestra bilingue & $\begin{array}{l}\text { Maestra } \\
\text { bilingue }\end{array}$ \\
\hline
\end{tabular}

Fuente: elaboración propia a partir de la información recogida en el trabajo de campo de la investigación.

Tabla 3. Características socioculturales de las mujeres entrevistadas

\begin{tabular}{|c|c|c|c|c|c|c|}
\hline 50 & Houston & Casada & 3 & $\begin{array}{c}\text { Licenciatura en } \\
\text { Idiomas }\end{array}$ & Maestra & Maestra bilingie \\
\hline 48 & Houston & Casada & 2 & $\begin{array}{l}\text { Master en } \\
\text { Educación }\end{array}$ & Traductora & Maestra bilingthe \\
\hline 56 & Houston & Casada & 2 & $\begin{array}{c}\text { Licenciatura en } \\
\text { Ciencias químicas }\end{array}$ & $\begin{array}{l}\text { Maestra } \\
\text { bilingue }\end{array}$ & Maestra bilingue \\
\hline 53 & Houston & Casada & 2 & $\begin{array}{l}\text { Cursando doctorado } \\
\text { en Educación }\end{array}$ & $\begin{array}{c}\text { Conserje en un } \\
\text { hotel }\end{array}$ & $\begin{array}{l}\text { Directora en una } \\
\text { escuela de primaria }\end{array}$ \\
\hline 55 & Houston & Separada & 1 & $\begin{array}{l}\text { Licenciatura en } \\
\text { Contaduria }\end{array}$ & $\begin{array}{l}\text { Maestra } \\
\text { bilingūe }\end{array}$ & Maestra bilingthe \\
\hline 23 & Monterrey & Soltera & 0 & Ingeniero & Au prair & $\begin{array}{l}\text { Trabaja como } \\
\text { ingeniero }\end{array}$ \\
\hline 33 & Monterrey & Casada & 2 & $\begin{array}{c}\text { Licenciatura en } \\
\text { Administración de } \\
\text { empresas }\end{array}$ & Au pair & $\begin{array}{c}\text { Trabaja en la } \\
\text { administración de } \\
\text { una empresa }\end{array}$ \\
\hline 30 & Monterrey & Soltera & 0 & $\begin{array}{l}\text { Licenciatura en } \\
\text { Nutrición }\end{array}$ & Mesera & Trabaja en nutrición \\
\hline 78 & Monterrey & Casada & 12 & $\begin{array}{l}\text { Fue a la escuela } \\
\text { hasta los } 12 \text { años }\end{array}$ & Jomalera & Ama de casa \\
\hline
\end{tabular}

Fuente: elaboración propia a partir de la información recogida en el trabajo de campo de la investigación. 
Antes de concluir este apartado necesitamos aclarar qué entendemos cuando nos referimos a la cualificación de las mujeres. Para ello, consideramos oportuno recurrir a dos definiciones, una elaborada por Steven Vertovec (2002) y la otra por el Instituto Nacional de Estadística y Geografía mexicano (INEGI). A partir de la primera definición se subrayan las habilidades poseídas por los individuos partiendo de un listado de profesiones que se vuelven expresión del nivel escolar:

[...] arquitectos, contables y financieros expertos, ingenieros, técnicos, investigadores, científicos, cocineros, maestros, profesionales de la salud, y - cada vez más - especialistas en tecnología de la información (TI, incluidos los profesionales de informática, ingenieros informáticos, directores, representantes de ventas. (VERTOVEC, 2002, p. 2)

En cambio, el Instituto Nacional de Estadística y Geografía mexicano (INEGI) se enfoca en la formación académica recibida por los y las profesionales que se definen como: "Los trabajadores que se caracterizan por haber recibido instrucción y formación profesional en el nivel de escolaridad superior: licenciatura, maestría, doctorado, posgrado o equivalente [...]" (Josefina GUZMÁN ACUÑA, 2013, p. 106).

La decisión de emplear ambas descripciones para detallar el concepto de cualificación se debe a la necesidad de representar la totalidad de un fenómeno que se distingue por características profesionales y académicas.

\section{La doble invisibilidad}

Según Pierrette Hondagneu-Sotelo (2007) el género y la clase social se vuelven aspectos clave en el análisis de las migraciones femeninas a medida que estos elementos permiten desafiar el androcentrismo que sigue caracterizando los estudios sobre las migraciones.

Siguiendo el ejemplo de esta autora, en el presente artículo utilizamos el análisis del género y la clase social para fundamentar la construcción teórica de los mecanismos que crean invisibilidad, cuyo resultado final es la formación de mano de obra femenina fácilmente explotable. Una condición que determina la inclusión de las mujeres migrantes en lo que Saskia Sassen (1984) define como un nuevo proletariado, que Monica Boyd (1984) llama underclass y que Douglas Massey (2014) denomina new urban underclass.

\section{De género}

Empezamos el análisis enfocándonos en la invisibilidad ligada al género, cuyo origen se relaciona al papel que la mujer realiza en el ámbito reproductivo, con la maternidad, y en el productivo, a partir del sistema de producción capitalista. La invisibilidad es, en este caso, directa consecuencia de dinámicas que mientras concurren a plasmar el ideal social de la mujer ama de casa, obscurecen su participación en el mercado laboral al subrayar su papel protagónico en el ámbito de la esfera reproductiva.

Ahora bien, para poder ahondar en estas dinámicas necesitamos volver a los estudios que las corrientes feministas llevan a cabo en la década de 1970. En esa época los intereses de las estudiosas se focalizan en la desigualdad de relaciones entre hombres y mujeres, así como en los mecanismos que desde el género desarrollan relaciones de poder, centrando el análisis en la lógica de dominación que subyace el sistema patriarcal y la economía capitalista. Se destacan así dos líneas principales de investigación, una enfocada en las relaciones de poder que se generan en el sistema patriarcal a partir del parentesco y el matrimonio (Kate MILLETT, 2000), y la otra focalizada en la sumisión de las mujeres a los hombres en el interior del sistema de producción capitalista (Lourdes BENERÍA, 1981). Así que, mientras en la primera se perfila un sistema de estratificación social en el cual las mujeres desarrollan un papel vehicular que se realiza a partir de alianzas matrimoniales mediante el intercambio de mujeres (Gayle RUBIN, 1986), en la segunda, el capitalismo incorpora las dinámicas del sistema patriarcal, favoreciendo el desarrollo de mecanismos de explotación que encuentran su expresión en la dicotomía público/privado (Samara DE LAS HERAS AGUILERA, 2009).

Centrando la atención en esta última línea de investigación, podemos observar que la división de los espacios según el género es el reflejo de dinámicas que acercan las mujeres a la naturaleza por su capacidad reproductiva y, por contraposición, los hombres a la cultura, estableciendo los ámbitos dentro de los cuales las funciones masculinas y femeninas se llevan a cabo. De tal manera que las mujeres operan dentro del espacio doméstico-privado y los hombres en el espacio público, con la consiguiente invisibilización de la participación femenina en la esfera económica. De estas consideraciones se desprende que la imagen de la mujer como principal proveedora de la reproducción de los hogares, por encarnar el papel de esposa y madre, minimiza su posición dentro del mercado laboral al caracterizar su participación en la economía familiar sólo como una ayuda al verdadero sustentador del núcleo doméstico, el hombre.

Este mecanismo se refleja, también, cuando analizamos la invisibilidad que acarrea la presencia de las mujeres en los flujos migratorios. A tal propósito, si nos acercamos a los datos 
estadísticos podemos observar que, según el Censo de Población mexicano, para el quinquenio 1990-1995 las mujeres representaban el 30\% de los migrantes, mientras que durante el periodo 1995-2000 la presencia femenina había bajado llegando al 25\%. Por el contrario, el Census Bureau de Estados Unidos para el periodo 1995-2000 registraba hasta el $42 \%$ de mujeres mexicanas en los flujos migratorios, presentando una diferencia de 17 puntos con los datos recogidos por la institución mexicana.

Hoy en día las cifras presentadas por las instituciones mexicanas se han acercado a los valores señalados por el instituto norteamericano, aunque sigue habiendo una brecha significativa entre las representaciones numéricas de ambos países. En 2013 el US Census Bureau señalaba la presencia de 11.581,977 migrantes procedentes de México, de los cuales 6.133,744 eran hombres y $5.451,233$ eran mujeres, representando cerca del $47 \%$ del total (Jesús A. CERVANTES GONZÁLEZ, 2015). En cambio, según el Instituto para Mexicanos en el Exterior, en 2016 el porcentaje de mujeres presentes en Estados Unidos correspondía al $41 \%$ frente al $59 \%$ de los hombres. ${ }^{4}$

Lo que podemos apreciar a partir de estos datos es que la construcción de la sobrerrepresentación de los varones en los movimientos migratorios con respecto a su contraparte femenina se produce también a nivel institucional. Un fenómeno que según Eleonore Kofman y Parvati Raghuram (2006) es consecuencia de la construcción de la imagen de la mujer dentro de la lógica de género, a partir de la cual la componente femenina de los desplazamientos adquiere un carácter pasivo por seguir al hombre en su recorrido migratorio sin conseguir insertarse en el mercado laboral.

\section{De clase}

De la invisibilidad de género pasamos a la invisibilidad de clase para mostrar el proceso que subyace la construcción de mano de obra barata y apta para la explotación (Sara SALVATORI; TERRÓN-CARO, 2017). Si la invisibilidad de género se relaciona con la supuesta ausencia de las mujeres del mercado de trabajo o de su presencia en sectores que se caracterizarían por ser "femeninos", la invisibilidad de clase tiene que ver con la ubicación de todos los/as migrantes mexicanos/as en una única clase social baja, ofuscando las diferencias económicas y culturales que caracterizan quienes emigran. Por este motivo, la noción de clase a la que nos referimos para poder observar el proceso de desclasamiento que se realiza al cruzar la frontera, es la que integra el aspecto económico al desarrollo de prácticas sociales dirigidas al consumo de determinados bienes. Así que, la posesión de capital económico y/o cultural son los elementos que determinan la posición social en un espacio estratificado (Pierre BOURDIEU, 1997). Cuando en el lugar de la migración estos elementos se invisibilizan lo que se produce es el aplanamiento de las diferencias, creando una población migrante ficticiamente homogénea que se inserta en los sectores no calificadas del mercado de trabajo. De hecho, Gandini y Lozano Ascencio (2012) señalan que los/ as migrantes mexicanos/as con grado de licenciatura se insertan en los mismos nichos laborales que las/os que tienen un bajo perfil escolar, mientras que las/os que cuentan con maestría y/o doctorado difícilmente llegan a desarrollar actividades ligadas a la formación recibida en el país de origen. Según la elaboración por parte de Luis Calva-Sánchez y Rafael Alarcón (2015) de los datos de la American Community Survey, entre 2009 y 2011 el 31,6\% del total de los migrantes procedentes de México eran migrantes cualificados/as, de los/as cuales el $20,7 \%$ realizaba una actividad técnica y el $47,6 \%$ trabajaba en sectores no calificados.

Ahora bien, si relacionamos la invisibilidad de clase al género podemos observar que el resultado es la sobrerrepresentación de las mujeres migrantes en determinados sectores laborales. En efecto, para el caso de las migraciones femeninas desde México, el alto número de mujeres que se emplea en el servicio doméstico, genera la errónea convicción de que los flujos migratorios femeninos están compuestos principalmente por migrantes de bajo perfil escolar. Al mismo tiempo, cuando la mirada recae en sectores calificados donde la concentración de varones es alta, como en el caso de las "finanzas y las ciencias y tecnología", se puede producir la convicción de que el porcentaje de hombres cualificados supera el de las mujeres (KOFMAN; RAGHURAM, 2006). Mientras que si nos fijamos en los pocos datos de los que disponemos, podemos observar que en 2011 las mujeres migrantes mexicanas con un alto nivel escolar representaban más de la mitad del total de los flujos migratorios cualificados, con 2.458 individuos equivalente a cerca del $52 \%$ del total (CALVA-SÁNCHEZ, 2014; RAMÍREZ GARCÍA; GANDINI, 2016).

\section{Consecuencias de la invisibilidad y su repercusión en el mercado de trabajo norteamericano}

Según cuanto observado en los apartados anteriores, las invisibilidades que se constituyen a partir del género y la clase determinan la inclusión de las mujeres en aquellos contextos laborales que, por un lado, corresponden a la división sexual de los roles y, por otro, concurren a la creación

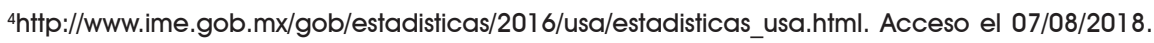


del prototipo de la mujer migrante. La representación que se genera en este último caso se relaciona a un modelo femenino originado a partir de un proceso de homogeneización hacia características que bajan el nivel sociocultural de las mujeres. Así que la inserción en el mercado laboral se realiza a partir de aquellos fenómenos que los diferentes autores definen como subutilización (LOZANO ASCENCIO; RAMÍREZ GARCÍA, 2015), brain waste o desperdicio de formación (Juan GONZÁLEZ BECERRIL, 2005), descualificación (Nina SORENSEN, 2005) y sobrecalificación, puesto que lo que se demanda es "el uso de sus manos y no de sus mentes", tal como señala Shahrzad Mojab (1999) al analizar la condición de las mujeres migrantes cualificadas en el mercado laboral canadiense. Del mismo modo se observa que: "En general, la mayoría de las migrantes comparten la experiencia de descualificación, es decir, que pierden su capacitación profesional" (SORENSEN, 2005, p. 163).

Desafortunadamente, para el caso mexicano, los pocos datos que se conocen acerca de la inserción de migrantes cualificados en el mercado laboral norteamericano no incluyen el enfoque de género, ubicando el análisis en un contexto asexuado (GONZÁLEZ BECERRIL, 2005; Adolfo ALBO; Juan L. ORDAZ DÍAZ, 2011 ; Selene GASPAR; Mónica CHÁVEZ, 2016). De todos modos, a pesar de la invisibilidad que la escasez de datos sigue generando alrededor de las mujeres migrantes cualificadas, disponemos de elementos que permiten acercarnos a las modalidades de inclusión en el mercado laboral de las y los mexicanos. A tal propósito, Albo y Ordáz (2011) muestran que México tiene tasas de migración cualificada superiores a las de India, Irán, Brasil y Colombia, menores a las de Vietnam y Cuba, y similares a las de Filipinas. Sin embargo, la inserción en el mercado laboral se lleva a cabo en sectores no calificados de la economía estadounidense. De la misma opinión es Rosa Emilia Bermúdez Rico (2010), quien observa que el $35 \%$ de los/as migrantes mexicanos/as residentes en Estados Unidos con grado de licenciatura ocupa puestos directivos, comparado con el $45 \%$ de los/as sudamericanos/as y el $64 \%$ de la población autóctona. En cambio, los/as migrantes mexicanos/as con maestría y/o doctorado en puestos directivos suman el $56 \%$ con respecto al $80 \%$ de los/as sudamericanos/as y al $86 \%$ de los/ as estadounidenses (Amba PANDE; TIGAU; Yan YUAN, 2017).

\title{
Análisis e interpretación de los resultados Inserción en el mercado laboral y descenso social
}

La escasez de datos acerca de las dinámicas que insertan las mujeres mexicanas cualificadas en empleos no calificados en el mercado laboral norteamericano nos obliga a incursionar en el aspecto narrativo de la investigación cualitativa para poder interpretar los mecanismos producidos por las invisibilidades de género y de clase. Invisibilidades que actúan al cruzar la frontera, cuando las mujeres cualificadas experimentan el descenso de la posición social.

En este sentido, Levitt y Glick Schiller (2004) observan que las/os migrantes que se desplazan para lograr mejorar su condición económica experimentan un descenso de la posición social al llegar a Estados Unidos, en la imposibilidad, o casi, de "transnacionalizar" la pertenencia a un determinado estatus social, sobre todo en relación a la migración de individuos con una alta formación escolar.

La mayoría de los testimonios recogidos corroboran estas reflexiones, evidenciando la formación de mecanismos enmarcados dentro de un sistema de género que, al ofuscar la presencia de las mujeres en el mercado laboral norteamericano, favorecen su explotación en el interior de sectores que se consideran de competencia femenina.

Así que, a pesar de contar con una licenciatura o un master, las mujeres se vuelven cuidadoras de ancianos, niñeras, camareras, etc., subrayando de este modo no sólo el "desperdicio" de la formación profesional adquirida en el lugar de origen, sino también la inserción en actividades ligadas a la división sexual de los roles.

\begin{abstract}
Cuando llegué a Houston empecé a tomar clases de inglés y un día una compañera de curso me dijo que tenía que dejar su antiguo trabajo, pues le habían ofrecido un contrato en otro, pero para no dejar sola la señora que cuidaba, se había ofrecido de buscar a alguien que tomara su lugar. Yo en ese momento tenía mi visa de turista y no necesitaba tener un trabajo formal, así que fui a conocer a la señora y decidí quedarme con ella. Este trabajo me gustaba porque tenía libres las mañanas y los domingos y me habían dado una habitación bien lujosa. Trabajaba en las tardes y las noches, la acompañaba en los restaurantes y hasta fui con ella dos veces a Europa. (BI, ${ }^{5}$ entrevista, 2009)

Tengo una licenciatura y una maestría en informática administrativa por la Universidad Autónoma de Nuevo León, pero cuando llegué a Houston al principio trabajé como camarera y cuidando niños, y ahora vendo publicidad para un periódico latino. (Ca1, entrevista, 2009)
\end{abstract}

${ }^{5}$ Se utilizan nombres ficticios para para salvaguardar el anonimato de las mujeres entrevistadas. 
Las dinámicas que se generan alrededor del género y la clase obtienen el efecto de deconstruir las características sociolaborales que forman parte de la vivencia que las mujeres han desarrollado en el país de origen. El cruce de la frontera en calidad de migrante conlleva un proceso que hemos definido como homogeneización hacia abajo para indicar la formación de un nuevo proletariado que se fundamenta a partir de dos fenómenos: 1) por un lado se encuentra lo que denominamos la deconstrucción sociolaboral del individuo, que se lleva a cabo a través del allanamiento de las diferencias sociales y educativas; 2) y por otro, la reubicación en un estrato social bajo tras la construcción de un nuevo perfil sociolaboral.

\section{Estrategias y desafíos}

La línea de investigación trazada hasta ahora, cuyo eje se encuentra en el análisis sociolaboral de los movimientos migratorios, nos impulsa también a plantear un análisis multisituado (MARKUS, 1995) bajo la concepción de la "multi-presencia" señalada por Levitt y Glick Schiller (2004) en relación con el fenómeno del transnacionalismo.

De este modo, es posible observar que cada acción genera múltiples efectos en relación con el contexto de inserción. La migración, de hecho, produce movimientos que, debido a las condiciones políticas y económicas de los lugares de origen y destino, modifica la posición social de los individuos, a pesar de las características socioeconómicas que forman parte del bagaje de los y las migrantes. Así que, si al cruzar la frontera con Estados Unidos las mujeres mexicanas con un alto nivel escolar se insertan en trabajos no calificados formando parte de la clase baja, en México siguen manteniendo una posición social adecuada a su nivel cultural y económico. Es más, la migración puede ser considerada como una estrategia para fortalecer la posición social en el país de origen (Mirjana MOROKVASIC, 2007).

Las crisis económicas de las décadas de los 80 y 90 del siglo pasado en México han determinado el desarrollo de trayectorias migratorias finalizadas al pago de las deudas contraídas a causa del desempleo o de la pérdida del poder económico de los salarios.

Decidí emigrar en 1995, cuando había perdido el trabajo y ya no alcanzaba para pagar la casa que estaba comprando, así que para no perderla me fui a Estados Unidos. Pues, en Houston trabajé de todo: estuve en manutención, limpiaba edificios y cocinaba hamburguesas en un restaurante. Llegué a tener hasta tres empleos a la vez para ganar más. (Sa, entrevista, 2011)

En otros casos, en cambio, asistimos al desarrollo de estrategias dirigidas hacia el reposicionamiento social en el lugar de la migración, a través de un proceso de movilidad social ascendente.

Tras terminar mis estudios en la Escuela Normal Superior empecé a trabajar como maestra de primaria en las escuelas públicas de Monterrey, hasta que a los 27 años me casé y decidí emigrar con mi esposo. En Houston trabajé en la limpieza, hasta que mis hijos entraron en la escuela, entonces me compré un carrito y comencé a manejar sin licencia para ir a trabajar como voluntaria en su escuela. Estuve colaborando con una de las maestras de mis hijos todos los días durante tres años, y me encontré muy bien pero nunca dije que yo también era maestra. Fue solamente cuando me preguntaron qué hacía en Monterrey, que les conté que era maestra de primaria. La maestra de mi hijo me ayudó dándome toda la información para empezar el curso para certificarme como enseñante también en Houston. Afortunadamente, mi esposo dos años antes había podido arreglar mis documentos y en 1998 me naturalicé norteamericana. [...] Lo más difícil fue aprender inglés. Me dolía el estómago porque todas las asignaturas eran en inglés. Tomé también un curso de escritura que duró seis meses. El maestro, un hombre moreno, africano, era bien duro y hablaba bien feo tratándonos como niños. Finalmente, después de dos años obtuve la certificación para trabajar como maestra. Poco a poco nuestra situación económica empezó a mejorar. Mi esposo tenía dos empleos en la mañana trabajaba en una tienda de video y en la noche limpiaba oficinas. Y cuando yo empecé a trabajar como maestra pudimos montar un negocio de construcción de casas flotantes para compañías petroleras. (Le, entrevista, 2009)

Cuando llegué [a Houston] y empecé a trabajar en ese hotel, en el restaurante, yo era la hostess, la cajera...fue cuando empecé a aprender inglés. Con el bebé chiquito fue una etapa difícil porque estábamos solos [ella y el esposo], yo trabajaba en la mañana y él en la tarde. Yo llegaba y él me pasaba la pelotita [el hijo recién nacido] y él se iba. Y luego él llegaba en la madrugada y yo me iba a las 5.30 de la mañana y yo le pasaba la pelotita. [...] Al rato me desanimaba porque decía: "Tengo mi carrera, mis estudios, trabajé en un periódico, entrevisté gente importante y aquí estoy de la nada." Pero luego yo decía: "Tengo que empezar de cero." Y mi esposo también me apoyaba porque cuando me veía llorando y desanimada me decía: "Pero, ¿no eras tú la que decía que sí se puede?" [...] En diciembre del 97 yo tenía mi certificado para trabajar como maestra. En el mismo distrito [donde estaba haciendo la práctica] me contrataron como maestra. Empecé como maestra bilingüe en primer grado y luego me cambié a otra escuela más cerca de la casa. Después me hice maestra de segundo grado y luego maestra de tercer grado. Pero cuando estaba maestra de tercer grado, el distrito mandó un email que ofrecían becas para maestría. Había que hacer una solicitud, ir a una entrevista 
porque había como 50 personas que solicitaban la beca y solamente iban a seleccionar a 12. Afortunadamente me dieron la beca e hice mi maestría en el 2008 en Educational direction. Terminé en diciembre de 2008 y la beca incluía una tesis. Durante ese tiempo yo nunca pensé en ser subdirectora, yo era feliz siendo maestra. Pero cuando empecé a estudiar, entonces te empiezan a meter el gusanito que puedes hacer más. Entonces dije: “iBueno!” En 2009 empecé a buscar que más había para mí y me hice especialista para ayudar a los maestros, tipo como coach. Estuve un año en esta nueva escuela y entonces empecé a sentir que podía ser assistant principal, subdirectora. Solicité así el puesto de subdirectora y empecé en 2010 y fue hasta junio de 2016. Este es mi primer año de directora. Cuando mi director se fue a otra escuela él me dijo: "Tú tienes que ser la directora aquí." Pero no dependía de él ni de mí, tenía que solicitar el puesto. Tuve que ir a entrevista, había mucha competencia, tuve que competir con otros, fui a la entrevista y gracias a Dios me dijeron que me daban el puesto. [...] Yo nunca pensé en ser maestra, yo cuando estaba en México quería ser abogada. Jamás pasó por mi mente, entonces creo que Dios es el que me ha guiado. Ser directora de una escuela, jamás lo hubiera pensado. Cuando me vine a los Estados Unidos, lo que quería era tener una zapatería, porque para mí el inglés siempre ha sido una barrera o un reto. Creo que esto que he logrado me ha costado mucho esfuerzo. Pero no es honor mío, yo no lo hubiera hecho sola, creo que Dios me puso aquí. Creo que con los estudios voy adquiriendo más cosas, y es cuando pienso que la gente que no tiene estudios, como los papás que vienen a registrar aquí sus hijos, su visión es bien limitada. A mí, por ejemplo, me limitaba el inglés a pesar de mis estudios en México. Hice una maestría y vi que podía hacer más y ahora que estoy haciendo el doctorado, y empecé como directora, yo digo que hasta aquí voy a llegar, pero no sé, porque con el doctorado a lo mejor se me va a abrir más la visión. [...]. (Ma16, entrevista, 2016)

Para poder entender las diferentes estrategias necesitamos enfocarnos en la finalidad del proyecto migratorio dirigiendo, así, la atención hacia la acción de las mujeres cualificadas. A tal propósito, en la tabla 4 mostramos la relación entre los objetivos de la migración y el desarrollo de estrategias finalizadas al fortalecimiento de la posición social en un contexto transnacional. En particular, a través de los objetivos que caracterizan el proyecto migratorio pretendemos subrayar la manera en que las mujeres migrantes actúan en relación con la movilidad social en la sociedad de origen y en la de asentamiento por medio también de estrategias ligadas a la socialización.

Tabla 4 - Relación entre los objetivos de la migración y el desarrollo de estrategias centradas en la posición social

\begin{tabular}{|c|l|l|}
\cline { 2 - 3 } \multicolumn{1}{c|}{} & \multicolumn{1}{c|}{ Objetivos de la migración } & \\
\cline { 2 - 3 } & Regresar a México & Quedarse en Estados Unidos \\
\hline Movilidad social & $\begin{array}{l}\text { Se refuerza la posición en el } \\
\text { lugar de origen }\end{array}$ & $\begin{array}{l}\text { Se refuerza la posición social en el } \\
\text { lugar de origen }\end{array}$ \\
\hline & $\begin{array}{l}\text { Desciende la posición social } \\
\text { en el lugar de la migración }\end{array}$ & $\begin{array}{l}\text { Se intenta ascender socialmente } \\
\text { también en el lugar de la migración }\end{array}$ \\
\hline $\begin{array}{c}\text { Estrategias ligadas a } \\
\text { la socialización }\end{array}$ & $\begin{array}{c}\text { Acercamiento a los } \\
\text { connacionales }\end{array}$ & Alejamiento de los connacionales \\
\hline
\end{tabular}

Fuente: Elaboración propia a partir de la información recogida en el trabajo de campo de la investigación.

Ahora bien, cabe especificar que para las mujeres cualificadas la migración crea un estado de incertidumbre que se relaciona a las posiciones socioeconómicas ocupadas simultáneamente en diferentes contextos geográficos. De tal manera que el proyecto migratorio acarrea consigo cuestionamientos tales como mantener la posición social en el país de origen o implementar medidas dirigidas al reposicionamiento social en el país meta de la migración.

A partir de estas premisas, como puede verse en la tabla 4, por un lado, se encuentran las mujeres que consideran la migración un periodo transitorio, después del cual volver a México. Pues, se trata de una migración que generalmente está focalizada al fortalecimiento de la posición socioeconómica en el país de origen. En este contexto, el periodo de tiempo limitado a la obtención de los ahorros facilita la aceptación del descenso social que se realiza en el país meta de la migración, y que se recompone una vez finalizada la experiencia migratoria. En cambio, las mujeres con una trayectoria migratoria que consideran definitiva intentan activar diferentes estrategias para poder ascender socialmente también en el lugar de asentamiento. 
Ahondando más en esta línea interpretativa, observamos que las mujeres que consideran temporal su estancia en Estados Unidos (Tabla 4) desarrollan estrategias de sociabilidad que traspasan las clases sociales, ajustándose a las condiciones del entorno en el que se insertan y con el objetivo de recrear lazos de ayuda mutua. Es así que hombres y mujeres mexicanos con diferentes características socioculturales crean un espacio común de socialización en el lugar de asentamiento sin importar la posición social. De este modo, consiguen construir redes de apoyo en un entorno que consideran hostil. Sin embargo, la vuelta al país de origen de las mujeres cualificadas, tras la obtención de los objetivos ligados al proyecto migratorio, permite retomar el estatus socioeconómico que se había ocupado antes de ir a Estados Unidos, reforzando la posición social y, al mismo tiempo, restableciendo las divisiones de clase.

Me juntaba con gente de México que no procedía de mi clase social, pero no me importaba, los veía cada fin de semana y hoy en día sigo visitándolos en Houston. [...] Decidí regresar a Monterrey cuando mi antigua jefa de trabajo me propuso un empleo muy ventajoso. (Sa, entrevista, 2011)

Cuando terminé la licenciatura en nutrición decidí ir a Indianápolis para mejorar mi inglés, así que me inscribí a un curso de idioma en la universidad y solicité la visa de estudiante. Para no depender totalmente del dinero que mis padres me enviaban, decidí trabajar en la cafetería de la universidad, pues era el único trabajo que podía hacer con mi visa. Sin embargo, cuando regresaba a casa después de cuatro horas de trabajo, lloraba porque tenía que estar parada todo el tiempo, barriendo, limpiando y trapeando, por 100 dólares semanales. [...] Cuando el curso de inglés se acabó, no quería volver todavía a Monterrey, así que me quedé en Indianápolis, aunque ya no tenía papeles, por este motivo me fui a la parte de la ciudad donde viven los latinos y empecé a trabajar en los bares nocturnos, donde sí tenía que aguantar los borrachos, pero ganaba 100 dólares la hora. [...] Después de un año, regresé a mi ciudad y ahora trabajo como nutricionista. (Ay, entrevista, 2010)

Cuando, en cambio, Estados Unidos se considera el lugar donde fijar la residencia, el deseo de ascensión social y profesional se une al intento de reconstruir aquella jerarquización que caracteriza la experiencia de las mujeres en el lugar de origen.

Una de las estrategias adoptadas en la comunidad de destino para reproducir el estatus social es el alejamiento de todo lo que recuerda su procedencia mexicana, considerada una desventaja en el ascenso social. Así que se eligen barrios fuera de los "guetos" mexicanos, se frecuentan iglesias con un bajo porcentaje de connacionales o se opta por seguir la misa en inglés, donde el número de mexicanos baja y, en cambio, sube la cantidad de coterráneos de clase medio-alta proclives a incorporarse a la sociedad receptora.

Yo vivo en un barrio donde hay básicamente norteamericanos y solamente dos o tres mexicanos. [...] No celebro ninguna fiesta mexicana, pero participo en los festejos de las norteamericanas [...]. Estoy metida en la organización de los ex alumnos del $\mathrm{Tec}^{7}$ de Monterrey y pertenezco a una iglesia cristiana. No voy a la misa en español porque los mexicanos que frecuentan esta iglesia son de otro estatus social, no son gente educada, son los que hacen trabajos humildes. ( $\mathrm{Ci}$, entrevista, 2011)

La distancia física subrayada por Ci se vuelve simbólica al rechazar cualquier cercanía con el estereotipo del mexicano. De este modo, la estrategia ligada a la socialización se fundamenta en el reposicionamiento sociocultural de las mujeres en la sociedad receptora.

\section{Conclusiones}

La invisibilidad que caracteriza la presencia de las mujeres mexicanas cualificadas en los flujos migratorios depende de mecanismos relacionados con el género y la clase social, cuyo resultado es la "desaparición" de las mujeres de las actividades productivas en los contextos internacionales. Sin embargo, cabe especificar que mientras la invisibilidad según el género se produce en el interior de un sistema que, para las diferentes corrientes feministas, se identifica con el sistema patriarcal y el sistema de producción capitalista, la invisibilidad según la clase es el producto de mecanismos que se articulan en el interior de las esferas económicas y políticas de los países receptores de mano de obra. En este contexto, la representación de la mujer como ama de casa se vuelve el elemento impulsor de su supuesta ausencia del mercado laboral y, por ende, de todas aquellas actividades relacionadas con la esfera productiva. Al mismo tiempo, la invisibilización de la clase social facilita la constitución de subgrupos colocados en posición marginal. De este modo, la descalificación social se refleja en la descalificación laboral que facilita la creación de mano de obra barata y sumisa a la precariedad de la oferta laboral en los sectores destinados a las mujeres mexicanas.

Estos procesos invisibilizadores generan lo que hemos denominado "homogeneización hacia abajo". En otras palabras, el descenso de la posición social determina mecanismos de homogeneización social ficticia, a través de los cuales, independientemente de las características 
socioculturales, todas las mujeres mexicanas se vuelven parte de una clase social baja en el lugar de la migración.

Ahora bien, la deconstrucción que afecta la posición social de las mujeres que migran debe leerse también a la luz del fenómeno transnacional. La presencia simultánea de las migrantes cualificadas en los lugares de origen y destino determina la posibilidad de mantener lazos económicos y sociales en ambos territorios, pero mientras en la comunidad de procedencia la ubicación social corresponde al capital social y cultural poseído, en el país meta de la migración esta conexión desvanece, determinando el descenso de la posición social. Un fenómeno que encuentra su respuesta en la lógica que subyace los sistemas productivos más proclives a atraer brazos que individuos.

Sin embargo, pese a los límites impuestos por el sistema, cabe señalar la capacidad de agencia de las mujeres cualificadas. A tal propósito, los datos recogidos durante el trabajo de campo han evidenciado como la finalidad del proyecto migratorio se vuelve el eje alrededor del cual se construyen estrategias de resistencia para que la experiencia migratoria sea conforme a las expectativas de las mujeres. De este modo, el descenso de la posición social determinado por mecanismos insertos en la macroestructura se reconfigura a través de la capacidad de acción femenina que se realiza simultáneamente en diferentes contextos geopolíticos.

\section{Bibliografía}

ALBO, Adolfo; ORDAZ DÍAZ, Juan L. Migración mexicana altamente calificada en EEUU y transferencia de México a Estados Unidos a través del gasto en la educación de los migrantes, 2011 . Disponible en: https://www.bbvaresearch.com/KETD/fbin/mult/WP_1125_Mexico_tcm346-266762.pdf.Acceso el 05/08/2018.

ARIAS, Patricia. "El viaje indefinido: la migración femenina a Estados Unidos". En: SÁNCHEZ, Martha J.; SERRA, Inmaculada (Eds.). Ellas se van. Mujeres migrantes en Estados Unidos y España. México: Instituto de Investigaciones Sociales de la Universidad Nacional Autónoma de México, 2013. p. 88128.

ARIZA, Marina. "Migration and family in Mexican research: a recent appraisal". Migraciones Internacionales, Tijuana, v. 7, n. 4, p. 9-37, julio/diciembre 2014.

BENERÍA, Lourdes. "Reproducción, producción y división sexual del trabajo". Mientras Tanto, Barcelona, n. 6, p. 47-84, 1981.

BERMÚDEZ RICO, Rosa Emilia. "Migración calificada e integración en las sociedades de destino". Sociedad y Economía, Cali, n. 19, p. 135-150, julio/diciembre 2010.

BOYD, Monica. "At a disadvantage: the occupational attainments of foreign born women in Canada". International Migration Review, New York, v. 18, n. 4, p. 1091-1119, winter 1984.

BOURDIEU, Pierre. Razones prácticas. Sobre la teoría de la acción. Barcelona: Editorial Anagrama, 1997.

BUSTAMANTE, Jorge A. "Migración de México a Estados Unidos: un enfoque sociológico". En: BUSTAMANTE, Jorge A.; TUIRÁN, Rodolfo (Eds.). La migración laboral mexicana a Estados Unidos de América: una perspectiva bilateral desde México. México: Instituto Matías Romero de Estudios Diplomáticos, SER, 1994. p. 25-72.

CALVA-SÁNCHEZ, Luis E.; ALARCÓN, Rafael. "La integración laboral precaria de los migrantes mexicanos calificados en Estados Unidos al inicio del siglo XXI". Papeles de Población, Ciudad de México, v. 21 , n. 83, p. 9-39, enero/marzo 2015.

CAPELLO, Carlo; CINGOLANI, Pietro; VIETTI, Francesco. Etnografie delle migrazioni. Roma: Carocci, 2014.

CERVANTES GONZÁLEZ, Jesús A. Migración, remesas y género: el caso de México. 2015. Disponible en: http://www.inegi.org.mx/eventos/2015/genero/doc/p_s6_JesusCervantes.pdf. Acceso el 05/08/ 2018.

CONAPO. La migración femenina mexicana a Estados Unidos. Tendencias actuales. 2013. Disponible en: http://www.conapo.gob.mx/work/models/OMI/Resource/652/1/images/ boletinMigracionNo1_8_03_13.pdf. Acceso el 05/08/2018.

DELGADO WISE, Raúl; CHÁVEZ ELORZA, Mónica Guadalupe; RODRÍGUEZ RAMíREZ, Héctor. "La innovación y la migración calificada en la encrucijada: reflexiones a partir de la experiencia 
mexicana". REMHU- Revista Interdisciplinar da Mobilidade Humana, Brasília, v. 24, n. 47, p. 153174 , mayo/agosto 2016.

DE LAS HERAS AGUILERA, Samara. "Una aproximación a las teorías feministas". Universitas. Revista de Filosofía, Derecho y Política, Madrid, n. 9, p. 45-82, 2009. Disponible en: http://universitas.idhbc.es/ n09/09-05.pdf. Acceso el 05/08/2018.

DE LILLO, Antonio (Ed.). II mondo della ricerca qualitativa. Torino: UTET, 2010.

FALZON, Mark A. Multi-sited Ethnography: Theory, Praxis and Locality in Contemporary Research. Great Britain: Ashgate Publishing. 2009.

GANDINI, Luciana; LOZANO ASCENCIO, Fernando. "La migración mexicana calificada en perspectiva comparada: el caso de los profesionistas con posgrado en Estados Unidos, 2001-2010". En: RAMÍREZ GARCÍA, Telésforo; CASTILLO, Manuel Ángel (Eds.). México ante los recientes desafíos de la migración internacional. Ciudad de México: CONAPO, 2012. p. 83-122. Disponible en: https://www.academia. edu/4413494/Gandini_Luciana_y_Fernando_Lozano_2012_La_migraci\%C3\%B3n_mexicana_cali ficada_en_perspectiva_comparada_èl_caso_de_los_profesionistas_con_posgrado_en_Estados_Unidos_20012010 ?auto=download. Acceso el 05/08/2018.

GASPAR, Selene; CHÁVEZ, Mónica. "Migración mexicana altamente calificada: 1990-2013". Problemas del desarrollo, v. 47, n. 185, p. 81-110, 2016. Disponible en: https://www.sciencedirect. com/science/article/pii/S0301703616300050. Acceso el 05/08/2018.

GONZÁLEZ BECERRIL, Juan G. "Inserción laboral de los migrantes calificados de origen mexicano en Estados Unidos, 1990-2000". Revista Argentina de Sociología, v. 3, n. 5, p. 88-106, 2005.

GUZMÁN ACUÑA, Josefina. "Mujeres profesionistas y mercados laborales: México y Tamaulipas". En: HERNÁNDEZ-HERNÁNDEZ Oscar M.; VERA VÁSQUEZ, Rodrigo (Eds.). Trabajo y género en Tamaulipas. México: El Colegio de Tamaulipas, 2013. p. 103-119. Disponible en :http://www.coltam.edu.mx/wpcontent/uploads/2014/04/Trabajo-y-G\%C3\%A9nero-en-Tamaulipas.pdf\#page=103. Acceso el 05/ $08 / 2018$.

HERNÁNDEZ-LEÓN, Rubén. Metropolitan migrants: the migration of urban Mexicans to the United States. Berkeley: University of California Press, 2008.

HONDAGNEU-SOTELO, Pierrette. "La incorporación del género a la migración: no sólo para feministas ni sólo para la familia". En: ARIZA, Marina; PORTES, Alejandro (Eds.). El país transnacional: migración mexicana y cambio social a través de la frontera. México: UAM-Juan Pablos Editor, 2007. p. 423452.

KOFMAN, Eleonore; RAGHURAN, Parvati. "Gender and global labour migrations: incorporating skilled workers." Antipode, Bruxelles, p. 282-303, 2006. Disponible en: https://s3.amazonaws.com/ academia.edu.documents/32180029/gender_and_global_labour_migrations.pdf?AWSAccessKey Id =AKIAIWOWYYGZ2Y53UL3A\&Expires $=153350-4870 \&$ Signature $=\mathrm{HJ}$ dhO\%2BQZOYKUJMyxG\%2FD4n TtB3R8\%3D\&response-content-disposition=inline\%3B\%20filename\%3DGender_and_Global_La bour_Migrations_Inco.pdf.Acceso el 05/08/2018.

LEVITT, Peggy; GLICK SCHILLER, Nina. "Conceptualizing simultaneity: a transnational social field perspective on society". International Migration Review, New York, v. 38, n. 3p. 1002-1039, septiembre 2004.

LOZANO ASCENCIO, Fernando; GANDINI, Luciana; RAMÍREZ-GARCÍA, Telésforo. "Devaluación del trabajo de posgraduados en México y migración internacional: los profesionistas en ciencia y tecnología". Migración y desarrollo, v. 13, n. 25, p. 61-89, julio/diciembre, 2015. Disponible en: http://www.scielo.org.mx/pdf/myd/v13n25/1870-7599-myd-13-25-00061 .pdf. Acceso el 05/08/2018.

LOZANO ASCENCIO, Fernando; RAMÍREZ GARCÍA, Telésforo. "Subutilización de las capacidades de los profesionales mexicanos de las ciencias y la tecnología y su vínculo con la migración los Estados Unidos". Notas de Población, Santiago de Chile, v.42, n. 101, p. 157-186, julio/diciembre 2015.

MARKUS, George E.. "Ethnography in/of the World System: the emergence of multi-sited ethnography". Annual Review of Anthropology, Palo Alto, n. 24, p. 95-117, 1995.

MASSEY, Douglas. "Manufacturing marginality among women and Latinos in neo-liberal America". Ethnic and Racial Studies, Surrey, v. 37, n. 10, p. 1747-1752, 2014. 
MILLETT, Kate. Sexual politics. USA: University of Illinois Press, 2000.

MOJAB, Shahrzad. "De-skilling immigrant women". Canadian Women Studies, Ontario, York University, v. 19, n. 3, p. 123-128, 1999. Disponible en: http://cws.journals.yorku.ca/index.php/cws/article/ viewFile/7886/7017. Acceso el 14/08/2017.

MOROKVASIC, Mirjana. "Migración, género y empoderamiento". Punto de Vista, Cuadernos del Observatorio de la Migraciones y de la Convivencia Intercultural de la Ciudad d Madrid, v. 3, n. 9, p. 33-49, 2007.

PANDE, Amba; TIGAU, Camelia; YUAN, Yan. "Skilled immigration and the conditions of labour competition in the US: a comparative study of the Indian, the Mexican and the Chinese diasporas". Migration and Development, v. 6, n. 3, p. 343-354, enero/marzo 2017.

PORTES, Alejandro. "Migración y desarrollo: una revisión conceptual de la evidencia”. Migración y Desarrollo, 2007. Disponible en: http://meme.phpwebhosting.com/ migracion/rimd/ coleccion_america_latina/migracionYdesarrollo/c1.pdf. Acceso el 05/08/2018.

RAMÍREZ-GARCÍA, Telésforo; GANDINI, Luciana. "Trabajadoras calificadas: las mujeres mexicanas en el mercado de trabajo estadounidense en perspectiva comparada". Revista Latinoamericana de Población, v. 10, n. 19, p. 33-56, julio/diciembre 2016. Disponible en: http://www.redalyc.org/ pdf/3238/323849595003.pdf. Acceso el 05/08/2018.

REBOLLEDO GÁMEZ, Teresa; RODRÍGUEZ CASADO, M. Rocío. "Migraciones y género en el contexto mexicano: revisión de la literatura científica". Foro de Educación, Salamanca, FahrenHouse, v. 12, n. 17, p. 165-185, 2014. Disponible en: http://dx.doi.org/10.14516/fde.2014.012.017.008. Acceso el 05/08/2018.

RUBIN, Gayle. "El tráfico de mujeres: notas sobre la "economía política" del sexo". Nueva Antropología, Ciudad de México, Universidad Nacional Autónoma de México, v. 8, n. 30, p. 95145 , noviembre 1986.

SALA, Emanuela. "L'intervista". En: DE LILLO, Antonio (Ed.). II mondo della ricerca qualitativa. Torino: UTET, 2010. p. 77-104.

SALVATORI, Sara; TERRÓN-CARO, Teresa. "Migrazione urbana-connessioni transnazionali. Elementi per una nuova interpretazione delle migrazioni messicane". Studi Emigrazione, Roma, n. 206, p. 307-323, aprile/giugno 2017.

SASSEN, Saskia. "Notes on the incorporation of third world women into wage-labor through immigration and off-shore production". International Migration Review, Ney York, Centre for Migration Studies, v. 18, n. 4, p. 1144-1167, winter 1984.

SOBRINO, Luis J. Migración urbana. La situación demográfica en México. México: CONAPO, 2010.

SORENSEN, Nina. "Migración, género y desarrollo: el caso dominicano". En: ZUiNPIGA GARCIA-FALCES, Nieves (ed.). La migracioìn, un camino entre el desarrollo y la cooperacioin. Madrid: Centro de Investigacioìn para la Paz, 2005. p. 163-182. Disponible en: http://www.fuhem.es/media/ecosocial/ file/Cohesión\%20Social/Inmigración/NYBERG\%20SORENSEN,\%20Ninna,\%20Migración,\%20género \%20y\%20desarrollo.pdf. Acceso el 05/08/2018.

STEPHEN, Lynn. "Towards a transborder perspective: U.S.-Mexico relations". Iberoamericana, Pittsburgh, v. 12 , n. 48 , p. $85-99$, diciembre 2012. Disponible en: http://www.iai.spk-berlin.de/fileadmin/ dokumentenbibliothek/lberoamericana/48-2012/48_Stephen.pdf. Acceso el 05/08/2018.

TERRÓN-CARO, Teresa; MONREAL GIMENO, María C. "Mujeres migrantes en tránsito en la Frontera Norte de México: motivaciones y expectativas socioeducativas ante el sueño americano". Papeles de Población, Ciudad de México, v. 20, n. 82, p. 128-166, octubre/diciembre 2014.

TIGAU, Camelia. "Skilled Mexican Migrants in Texas: What the Numbers Hide". México Center. Institute for Public Policy of Rice University, Julio 2017. Disponible en: https://scholarship.rice.edu/bitstream/ handle/1911/98848/MEX-pub-Migrants-071017.pdf?sequence=1. Acceso el 05/08/2018.

VERTOVEC, Steven. "Transnational networks and skilled labour migration". 2002. Disponible en: http://www.transcomm.ox.ac.uk/working\%20papers/WPTC-02-02\%20Vertovec.pdf. Acceso el 05/08/ 2018. 
WOO MORALES, Ofelia. "La migración de las mujeres mexicanas hacia Estados Unidos". Revista Población y Desarrollo, Paraguay, n. 10, p. 55-68, 2014. Disponible en: http://www.lamjol.info/ index.php/PDAC/article/view/1738. Acceso el 05/08/2018.

Sara Salvatori (sarasalvatori@hotmail.com) es Doctora por la Facultad de Ciencias Sociales de la Universidad Pablo de Olavide (Sevilla, España), Master en Políticas del Encuentro y Mediación cultural por la Università degli Studi Roma Tre (Roma, Italia), Licenciatura en Antropología Cultural por la Sapienza Università di Roma (Roma, Italia). Sus principales líneas de investigación se centran en: Migraciones transnacionales; Enfoque interseccional; Migraciones cualificadas; Género. Ha presentado ponencias en congresos internacionales en diferentes países y ha desarrollado trabajo de campo en Italia, España, Marruecos, México, Estados Unidos y Chile. En 2008 ha sido ganadora del primer premio en el ámbito del IX Premio Santo Padre Rubio S. J. para avances en el conocimiento de la inmigración, convocado por el Instituto Universitario sobre Migraciones de la Universidad Pontificia Comillas (Madrid, España).

Teresa Terrón-Caro (mttercar@upo.es) es Doctora del Departamento de Educación y Psicología Social de la Facultad de Ciencias Sociales, Universidad Pablo de Olavide (Sevilla, España). Doctora en Pedagogía por la Universidad de Sevilla. Co-directora del Máster Oficial en Género e Igualdad de dicha universidad y Directora del Grupo de Investigación en Acción Socioeducativa. Entre sus principales líneas de investigación se encuentran: Migraciones internacionales, Género y Educación; Diversidad Cultural e Inclusión educativa. Ha sido investigadora del proyecto Gender and Citizenship (GENDERCIT) (Referencia: PIRSES-GA-2012318960) Entidad financiadora y convocatoria: EUROPEAN UNION. SEVENTH FRAMEWORK PROGRAMME Marie Curie Actions (FP7-PEOPLE-2012-IRSES - No 318960) y actualmente forma parte del Proyecto de investigación La Educación Infantil en Alemania, Francia, Inglaterra, Suecia y España: Conciliación del Ámbito Familiar y Laboral del Plan Estatal 2013-2016 Retos - Proyectos I+D+i (EDU2016-78134-R) (2016-2019).

\section{COMO CITAR ESSE ARTIGO DE ACORDO COM AS NORMAS DA REVISTA}

SALVATORI, Sara; TERRÓN-CARO, Teresa. "La migración invisible de las mujeres mexicanas cualificadas". Revista Estudos Feministas, Florianópolis, v. 27, n. 2, e54443, 2019.

\section{CONTRIBUIÇÃO DE AUTORIA}

Sara Salvatori: Concepção, Coleta de dados, Análise de dados.

Sara Salvatori y Teresa Terrón-Caro: Elaboração do manuscrito, revisão e aprovação da versão final do trabalho.

\section{FINANCIAMENTO}

Não se aplica

CONSENTIMENTO DE USO DE IMAGEM

Não se aplica

APROVAÇÃO DE COMITÊ DE ÉTICA EM PESQUISA

Não se aplica

\section{CONFLITO DE INTERESSES}

Não se aplica

\section{LICENÇA DE USO}

Este artigo está licenciado sob a Licença Creative Commons CC-BY Internacional. Com essa licença você pode compartilhar, adaptar, criar para qualquer fim, desde que atribua a autoria da obra. 


\section{HISTÓRICO}

\section{Recebido em 08/12/2017}

Reapresentado em 07/08/2018

Reapresentado em 15/10/2018

Aprovado em 16/10/2018 